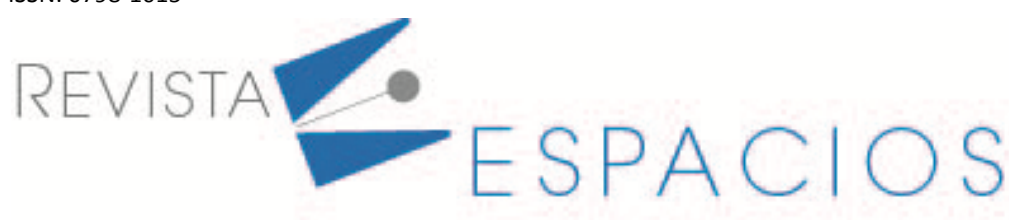

Vol. 41 (50) $2020 \cdot$ Art. 3

\title{
Creación de valor en la organización a partir de la estrategia del data influencer
}

\author{
Value creation in the organization from the data influencer strategy
}

\author{
MARCELINO-ARANDA, Mariana ${ }^{1^{*}}$ \\ PÉREZ CABRERA, Delia E. ${ }^{2}$ \\ TORRES-RAMÍREZ, Aylin ${ }^{3}$ \\ CAMACHO, Alejandro D. ${ }^{4 *}$
}

\begin{abstract}
Resumen
Se estructura un marco conceptual del Data Influencer para su reconocimiento como estrategia del Influencer Marketing. La revisión de la literatura fue de 2009 a 2019. Se muestran casos de éxito en la creación de valor de las partes interesadas. La confianza en las fuentes externas para extraer valor, plataforma, tecnologías disponibles y costos son los principales retos de las organizaciones para integrar estas estrategias en sus modelos de negocio. Se tiene una nueva generación de influencers de la plataforma Tiktok.

Palabras clave: creación de valor, modelos de negocio, influencer marketing, inteligencia de negocios.
\end{abstract}

\begin{abstract}
A conceptual framework of Data Influencer is structured to be recognized as a strategy of Influencer Marketing. The literature review was from 2009 to 2019. Successful cases of stakeholder value creation are shown. Relying on external sources to extract value, platform, available technologies, and costs are the main challenges of organizations that seek to integrate these strategies into their business models. There is a new influencers generation on the Tiktok platform.
\end{abstract}

key words: value creation, business models, influencer marketing, business intelligence.

\section{Introducción}

En el ámbito empresarial, los avances tecnológicos e internet generan nuevos modelos de negocios y con esto, estrategias que crean y capturan valor para las partes interesadas, además de generar ventaja competitiva (Zott y Amit, 2009). Algunas estrategias se concentran en el marketing, por medio de sus ejes principales: canales de comunicación, distribución y servicios (Baber et al., 2016; Kotler y Keller, 2012). Asimismo, el desarrollo de la Inteligencia de Negocios (en adelante, por sus siglas en inglés, BI) y el Análisis de Impacto de Negocio (en adelante, por sus siglas en inglés, BIA) ocasionó que las organizaciones comenzaran a extraer el conocimiento, manipular y procesar los datos estructurados y no estructurados que se generan en su ámbito de influencia, a

\footnotetext{
${ }^{1}$ Doctora en Ciencias Administrativas. Profesor Investigador. Instituto Politécnico Nacional. E-mail: mmarcelino@ipn.mx. *Autor de correspondencia

${ }^{2}$ Egresada de la Licenciatura Ciencias de la Informática. Instituto Politécnico Nacional. E-mail: pcedelia@gmail.com

${ }^{3}$ Maestra en Administración. Instituto Politécnico Nacional. Universidad Autónoma Metropolitana. E-mail: tor.ram.a12@gmail.com

${ }^{4}$ Doctor en Ecología Química. Profesor Investigador. Instituto Politécnico Nacional. E-mail: adcamachov@yahoo.com.mx. *Autor de correspondencia
} 
través de técnicas como el Big Data, Data Mining, Inteligencia Artificial y Deep Learning (Saura y Bennett, 2019). Asimismo, por adquirir el valor comercial de los datos. Es decir, las decisiones que toma la alta gerencia son resultado de la alineación de iniciativas y procesos estratégicos de fuentes externas de datos, que figuran como portadoras de valor, lo que mejora aspectos de innovación y rendimiento empresarial (Božič y Dimovski, 2019; Muntean, Cabău, y Rînciog, 2014).

Las fuentes externas se clasifican a través de dos corrientes principales: la Voz del Consumidor (en adelante VoC), conformada por las opiniones generadas sobre un producto o servicio y asociadas, principalmente, a la experiencia de consumo; y la Voz del Mercado (en adelante VoM) que se refiere a las tendencias que se presentan de acuerdo con las condiciones de los consumidores (Berlanga et al., 2015).

Las principales fuentes externas que ofrece un canal bidireccional entre la empresa y los consumidores son los medios y redes sociales (Sukrat y Papasratorn, 2018). La popularidad de medios sociales como Blogs, Wikis, Foros y redes como Facebook, Twitter e Instagram, se debe a su capacidad de difusión (Palacios-Marqués et al., 2015), alto nivel de interacción y disponibilidad (Baber et al., 2016). De hecho, las redes sociales se benefician de los últimos avances en tecnología por medio de los sistemas de información Web, principalmente Web 2.0 o Web Social. Esta Web permite al usuario generar contenido como comentarios, opiniones, expresiones e interacciones (Saura y Bennett, 2019). La Web 3.0, para el caso del BI y BIA, está relacionada con el Linked Open Data. Es decir, se refiere a datos vinculados mediante semántica de interpretación, que permite el uso de técnicas para la extracción y procesamiento de datos con el propósito de facilitar la toma de decisiones.

El principal reto al que se enfrentan las estrategias de negocio basadas en $\mathrm{BI}$ y BIA es el establecimiento de planteamientos multidimensionales. Los cuales, presentan bajo desempeño en los resultados al ser modelados mediante métodos de pronóstico tradicionales como la regresión lineal y las series de tiempo (Cui, Gallino, Moreno, y Zhang, 2018). Po lo que, las estrategias deben centrarse en características específicas de alcance, escala, velocidad (Bharadwaj, Sawy, Pavlou, y Venkatraman, 2013) y en la particularidad de cada técnica. Por ejemplo, en el Big Data, se debe tomar en cuenta el volumen, la variedad, la veracidad y el valor (Yuan, $\mathrm{Xu}, \mathrm{Li}, \mathrm{Y}$ Lau, 2018). Al respecto, las empresas se han inclinado por la generación del Management knowledge sobre la VoC y la VoM, ya que esto representa mayor probabilidad de que los usuarios manifiesten de forma implícita sus preferencias mediante el contenido generado en estos canales (Tsutsumi y Silva, 2019).

\subsection{El papel de la estrategia para la estimulación de fuentes externas}

La empresa es sostenible a partir de los recursos estratégicos que crea y ejecuta. La estrategia es la que logra crear una posición única en el mercado (Porter, 1996). Es decir, al momento de identificar, desarrollar, y adaptar sus recursos, la empresa obtiene ventaja competitiva y es sostenible en la medida en que cuenta con la capacidad de controlar esos ciclos vitales (Teece, 2007). Las estrategias comerciales son el medio para obtener valor en el tráfico de clientes y su fidelización. Además, a través de éstas la empresa busca fortalecer la confianza en sus productos, incentivar la intención de compra del consumidor, contribuir a la conciencia y reputación de la marca para generar una rentabilidad y posición sostenible (Saura y Bennett, 2019; Sivarajah, Irani, Gupta, y Mahroof, 2019).

Las preferencias o intención de compra expresadas por las personas se ven influenciados por diversos factores individuales como son el contexto demográfico, la cultura, la rutina, los gustos, las opiniones, los eventos y las situaciones diarias (Tsutsumi y Silva, 2019). Factores de la empresa que permiten incrementar preferencias y ganar la confianza del consumidor son: la imagen corporativa, la marca, la reputación, el tamaño, la fiabilidad y la seguridad (Sukrat y Papasratorn, 2018).

Como resultado de los esfuerzos de las organizaciones para generar interacciones entre las partes interesadas, aparecen las campañas de marketing Word of Mouth (WOM). El WOM tiene el propósito de promover la 
generación de comentarios, generalmente positivos, entre comunidades de negocio, expresados por los consumidores a través de medios y redes sociales en su rol de usuarios (Petrescu, O'Leary, Goldring, y Ben Mrad, 2018; Baber et al., 2016). Sin embargo, también existe Negative Word of Mouth (NWOM) que tiene el objetivo de dañar la reputación de un producto o servicio y que, en casos más complejos, las quejas, pueden afectar completamente a la marca (Balaji, Khong, y Chong, 2016; Haryani y Motwani, 2015).

Este tipo de campañas, en particular, se crean para influir en el comportamiento del consumidor de forma indirecta, ya que las organizaciones no están ligadas con la forma de transmisión, ni con el contenido del mensaje que los consumidores emiten, (Zengin y Gündüz, 2018) lo que le da mayor credibilidad (Li y Du, 2011). Un ejemplo de los esfuerzos para la difusión de comentarios por parte de los consumidores es la aplicación de la forma digital del WOM, el Electronic Word of Mouth (eWOM), donde las marcas buscan persuadir al consumidor a través de la generación de comentarios entre su círculo social mediante las plataformas de Internet (Petrescu et al., 2018).

Existen dos principales razones por las que los consumidores recurren a las opiniones generadas entre comunidades. Porque buscan información de manera confiable sobre algún producto o servicio y la calidad de éste. Y porque no cuenta con la suficiente información de la marca y de lo que se busca adquirir, ya sea por omisión de la empresa o por ser un producto de origen extranjero (Ren, Yeoh, Shan Ee, y Popovič, 2018; Baber et al., 2016). Sin embargo, cuando el usuario crea contenido y lo comparte, se genera una enorme cantidad de datos que en la mayoría del tiempo no son revisados. La generación de mayor cantidad de contenido no significa más calidad en los datos (Palacios-Marqués et al., 2015). Por lo que esto se convierte en uno de los principales retos para el análisis de la información de las estrategias a emplear por parte de las organizaciones.

\subsection{Influencer marketing}

Con mejor posicionamiento en las últimas dos décadas, dentro de las acciones del WOM se encuentra el Influencer Marketing (en adelante IMKT) que, en comparación con vídeos virales, desafíos y otras herramientas para llegar al consumidor, puede verse como una interacción directa de consumidor a consumidor, lo que genera más confianza para llevar a cabo la compra de productos o servicios (Petrescu et al., 2018; Baber et al., 2016; Zhu, 2013). El IMKT son aquellas actividades de marketing que se realizan a partir de las características de una persona y no de un segmento de mercado. Es decir, el alcance de la influencia de la persona va a definir el tipo de mercado al que se quiere llegar (Bamakan, Nurgaliev, y Qu, 2019). Por lo que, la identificación del perfil de las personas a las que se planea integrar en este tipo de campañas es el paso principal para el éxito de esta actividad.

La influencia social es factor clave para la difusión de ideas y la inducción de comportamientos. Lo cual, altera la forma en que los consumidores perciben la información y el valor que le asignan (Zengin y Gündüz, 2018). El objetivo de un Influencer es expresar el valor de la experiencia y utilidad de un producto o servicio consumido (Lin, Bruning, y Swarna, 2018). Este valor es lo que generara apego a una intención de adquisición por parte del consumidor (Bamakan et al., 2019).

Existen diferentes tipos de personas que están involucradas en el IMKT. Se identifican al prescriptor de una marca, quién es una persona con determinada fama en la actividad que desempeña. Por ejemplo, un jugador de fútbol o una actriz, quienes, al hacer promoción sobre alguna marca, no necesariamente deben tener conocimiento en el tema. Su influencia se basa en su imagen y la credibilidad o el público al que llega el anuncio (Gómez Nieto, 2018).

El líder de opinión es aquella persona capaz de ejercer influencia con gran impacto sobre la opinión de un tercero hasta el punto de darle una nueva estructura a la idea (Li y Du, 2011). Este término fue utilizado desde 1950 por distintas disciplinas con el objetivo de identificar al personaje influyente de las cadenas de influencia, donde no solo se contemplan las cualidades individuales como las fisiológicas, sociales, credibilidad, económicas, etc., sino también las del grupo en general, donde al líder de opinión se le percibe por arriba del promedio (Lin et al., 2018; 
Li y Du, 2011). Al respecto, Bamakan et al. (2019) menciona los factores que determinan el tipo de líder: alcance de la influencia, dominio del conocimiento, comportamiento y tiempo (Figura 1). Por ejemplo, en el factor dominio del conocimiento se encuentran los influencers monomórficos, con la emisión de opiniones con base en su tema de especialidad, y los polimórficos que tienen conocimiento de diversas áreas.

Figura 1

Categorización de líderes de opinión

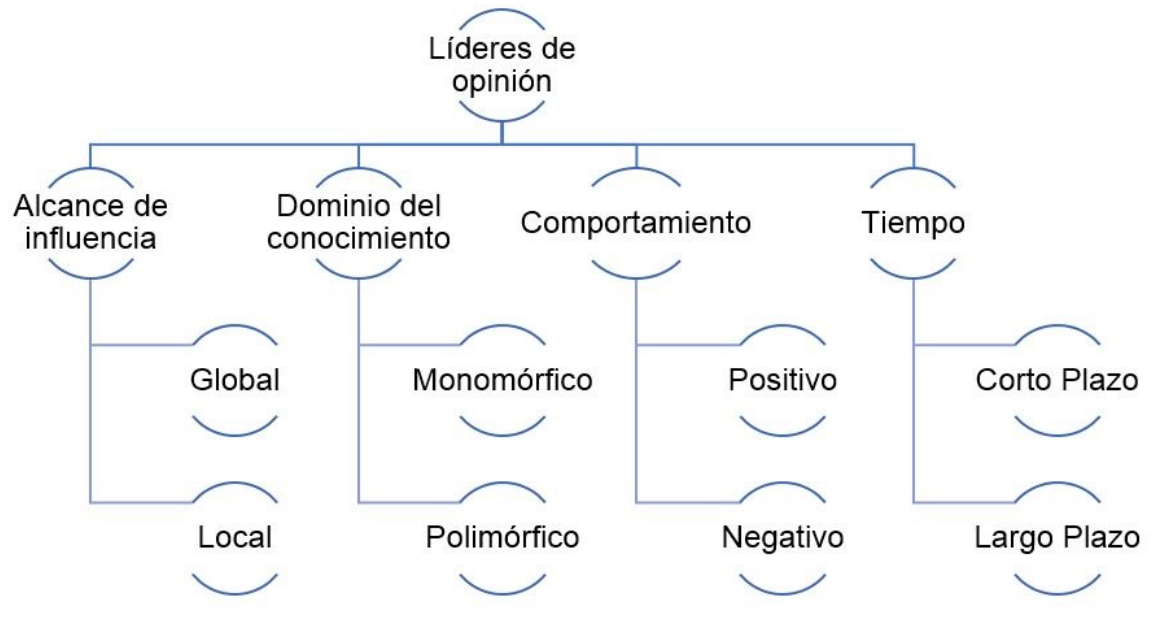

Fuente: información obtenida de Bamakan et al. (2019)

Una tercera persona fundamental en el IMKT es el influencer. Éste se considera como la evolución digital del líder de opinión, pues comparten objetivos en común además de que ambos cuentan con un nivel de influencia sobre otras personas. Esta presencia (sobre todo en las redes sociales) unida con la confianza que tienen los seguidores sobre algún tema determinado, pueden a la vez convertirlo en un prescriptor estratégico de marca (Gómez Nieto, 2018). La principal diferencia del influencer con los demás términos involucrados en la estrategia de marketing es la cadena de influencia que puede ejercer mediante su capacidad de interacción y contenido generado. Su presencia se mide a través de parámetros como la difusión e influencia. Sin embargo, estos parámetros muestran escasa atención en la confianza, la cual, es un factor fundamental para aludir las decisiones de compra de los consumidores. En este sentido, para que un consumidor pueda ser influenciado debe tener un nivel de confianza hacia el influencer y con ello se logre que la recomendación cambie la intención de compra (Zhu, 2013).

Los influencers con base en la confianza que emiten se clasifican en tres tipos. Los emerging influencers son personas que cuentan con un nivel de confianza bajo y están en la etapa inicial de su influencia, por lo que su alcance apenas se encuentra en expansión. Los holdings influencers se caracterizan por tener un nivel de confianza alto y cuentan con un alcance estable, aunque su crecimiento es lento. Por último, los vanishing influencers muestran una influencia en decadencia debido a la falta de interacción o alteraciones en su reputación (Liu et al., 2015; Zhu, 2013).

EI IMKT busca llegar a grandes volúmenes de personas a bajo costo, a través de su activo principal, el influencer. Por lo que, uno de los retos de las campañas de IMKT es buscar al influencer más conveniente para influir informalmente en las actitudes o comportamiento de los consumidores. Para lo cual, se tiene el siguiente procedimiento: 1) establecer el objetivo de la influencia a ejercer, 2) identificar a los influencers que se apeguen al contexto de la marca, y considerar su impacto y alcance, 3) seleccionar a los que concuerden con el objetivo de la campaña, 4) atraer e incentivar la colaboración entre la marca y el influencer, 5) coordinar esfuerzos de los involucrados para lograr el objetivo y 6) medir resultados con apoyo de BI y BIA mediante indicadores de confianza (Uzunoğlu y Misci, 2014; Li y Du, 2011). 
Se espera que en los próximos años el crecimiento de los usuarios de plataformas sociales en Internet ascienda aproximadamente a $1 / 3$ de la población mundial (Sharma, Ahuja, y Alavi, 2018). Por lo que, el desarrollo e implementación del IMKT para mejorar las estrategias de comercialización de las organizaciones mediante la identificación de patrones de comportamiento, se consideran de gran importancia, toda vez que incrementan la creación de valor para las partes interesadas. Es así como el objetivo de este trabajo es estructurar un marco conceptual del Data Influencer para su reconocimiento como estrategia del IMKT en la creación de valor en las organizaciones

\section{Metodología}

Se realizó una investigación documental a partir del cual, se buscó analizar las publicaciones de alto impacto sobre el tema y presentar una base fundamental de conocimiento. Las fuentes de información documental utilizadas fueron bibliográficas, hemerográficas y cibernéticas. Se emplearon guías de análisis documental, que sirvieron como instrumento para organizar e identificar los distintos conceptos que serían sometidos a revisión.

La revisión y análisis de la literatura en fuentes bibliográficas y hemerográficas se llevó a cabo del periodo de 2009 a 2019 en la colección principal del portal Web of Science. La colección principal almacena fuentes de información líderes en el ámbito académico y de investigación, como son el European Journal of Operational Research, Industrial Marketing Management, o el Journal of Enterprise Information Managemen. Asimismo, se utilizó la base de datos de Scopus. El uso de guías de análisis documental permitió obtener datos en dos fases. La primera, orientada a los elementos y factores que dan soporte al IMKT, los actores involucrados y las plataformas donde se desarrolla. Los temas de búsqueda fueron: Opinion leader/ Customer behavior / Influencer Marketing / Social Network. La segunda fase estuvo orientada a las fuentes de donde se obtienen los datos procesados por las empresas mediante herramientas de BI para la toma de decisiones y los elementos necesarios que componen la estrategia tecnológica. Para lo cual, se establecieron los siguientes temas de búsqueda: Business Inteligence / Opinion Mining / Marketing Intelligenece / Social Business Inteligence. Los datos obtenidos se almacenaron en una matriz de información, creada en programa Excel.

Para estudiar el comportamiento de usuarios de la red y la presencia de los influencers, particularmente en México, se usaron fuentes cibernéticas como las publicaciones del Digital Report, los informes de eventos como los Eliot Awards 2018, 2019 y 2020, plataformas de rankings como Hootkit, las publicaciones de 2018 a 2020 de páginas oficiales como YouTube, Facebook, Instagram y Twitter; los informes "Magic Cuadrant for Analytics and Business Intelligence Platforms" emitidos de 2018-2020 por la Consultora Gartner; y visitas a plataformas y portales en la web como SocialBlade, Coobis, Influencity e Internet República.

\section{Resultados}

\subsection{Implementación de $\mathrm{BI}$ en fuentes internas}

A través de los informes "Magic Cuadrant for Analytics and Business Intelligence Platforms" emitidos en el período de 2018-2020 por la Consultora Gartner, se observa que las herramientas de Tableu y Qlik son constantes en su liderazgo frente a otras que permanecen ubicadas en los cuadrantes de aspirantes, visionarios y expertos de nicho. A continuación, se presentan algunos ejemplos del uso y beneficio de estas herramientas en las empresas.

Hello Fresh implementó Tableau como una herramienta especializada en la optimización de tiempo para la generación de reportes internos de marketing. Su aplicación permitió la visualización y el rastreo de datos en tiempo real que apoyan la toma de decisiones con base en el comportamiento de los clientes y la optimización de las campañas de marketing. Se logró el aumento en las tasas de conversión y retención de clientes. 
Aeroméxico implementó la consistencia de datos a los formatos de fuentes internas y externas. Se logró que la información estuviera unificada en un solo sistema, lo cual permitió detectar los errores con rapidez y aprovechar la visualización dinámica para observar las tendencias (Tableau, 2019).

Por su parte, Qlik brinda sus servicios de BI a grandes empresas. Planet Hollywood, la cadena de restaurantes temáticos con carácter internacional, logró identificar las tendencias y patrones que le permiten optimizar las operaciones de sus distintas ubicaciones. La compañía Lenovo se apoyó en el análisis de datos consolidados de Salesforce y SAP. Deloitte sustituyó los informes mensuales por una aplicación de análisis de preferencias de consumo. Sony Europe implementó la herramienta QlikView y logró una solución integral para las operaciones del comité ejecutivo y las áreas de finanzas, marketing, operaciones, servicio y soporte, y cadena de suministro (Qlik, 2019).

\subsection{Propuesta de datos para la extracción de valor en fuentes externas}

El origen y desarrollo del IMKT se da a través del internet. Los seguidores tienen interacción con los influencers y fungen como el canal de comunicación entre los usuarios, a través de las plataformas sociales (Baber et al., 2016). Medir el impacto que tiene el influencer en un mercado específico y en la población virtual en general, requiere asociar su alcance con las cifras de usuarios. A través de plataformas de analítica se da seguimiento a las métricas de rendimiento de las redes sociales propias de la empresa y del impacto de las campañas. También hay indicadores para evaluar el desempeño del influencer (KPI), los cuales, permiten proyectar el alcance que tiene su participación en una campaña. Los resultados de estos impactos, compras por parte del consumidor, incentivan a las organizaciones a utilizar estrategias asociadas al IMKT.

Existen distintas formas de establecer indicadores orientados a cumplir los objetivos de una campaña a través de la colaboración entre el influencer y la empresa. Algunas plataformas de analítica proporcionan datos de forma gratuita, como es el número de suscriptores. Este número también se obtiene de indicadores que se encuentran en los perfiles de cada red social, menciones que se tienen de las publicaciones en un período de tiempo en redes sociales, reacciones a sus publicaciones, si se establece un "trending topic", entre otros.

El tamaño de la comunidad influenciada se analiza de forma general por zona geográfica, por demografía y de forma específica por el perfilamiento de preferencias y tendencias de consumo. Esta información del usuario digital puede ser tomada de sus búsquedas, de sus sitios frecuentes de visitas y las aplicaciones que utiliza. Por ejemplo, empresas como Amazon, Facebook, Mercado Libre, Google, entre otras plataformas, acceden a estos datos para ofrecer sugerencias de compra al consumidor. Para perfilar una comunidad se debe considerar su contexto digital. Existen organismos privados y públicos que emiten informes descriptivos del uso de internet. Po ejemplo, de forma global, a través del informe Digital Report publicado por We Are Social (Simon, 2019) se obtienen los siguientes datos:

- Existen 4,39 billones de usuarios conectados a la red en la escala global.

- El número de usuarios se ha incrementado en 2019 en 9\% respecto al año 2018.

- Se estima que los usuarios pasan en línea en promedio 6 horas y 40 minutos al día.

- Existen 3.48 mil millones de personas que usan las redes sociales y le dedican alrededor de 2 horas y 16 minutos. Lo que representa aproximadamente un tercio del tiempo activo del día otorgado a permanecer online e indagar en las redes.

En México, las cifras presentadas en el reporte Digital 2019 (We Are Social, 2019) informan que:

- Existen 88 millones de usuarios conectados, los cuales al menos están asociados con una red social.

- Se estima que pasan en promedio más de 3 horas en redes sociales. 
- El ranking de las plataformas sociales muestra entre los primeros lugares de popularidad a YouTube con una actividad del $95 \%$, Facebook con $93 \%$, Instagram con $64 \%$ y Twitter con $57 \%$.

A partir de los datos anteriores, resulte interesante identificar y analizar los influencers más valorados por la población mexicana, así como su presencia en las principales redes sociales utilizadas en el país. Esto permite conocer el alcance de su influencia por medio del número de seguidores y las áreas en las que se establecen estos personajes. Con esta información, las empresas podrían preguntarse ¿a qué segmento de los 88 millones de usuarios podrían llegar?, de las tres horas en promedio que los usuarios pasan en redes sociales ¿cuánto tiempo las empresas pueden percibir su atención sin ser invasiva?, y ¿cuál es el mejor canal para acercarse que se ajuste a los objetivos del análisis y posteriormente de la campaña?

Para los fines de esta investigación, se tomó como indicador "estatus de los influencers". El cual, es el reconocimiento que reúne a los líderes digitales más importantes en la industria de contenido y medios digitales de habla hispana. Los Eliot Awards (patrocinados por empresas como Andrea, Ford, Pañuelos Kleenex, Visit México y Passport Scotch) aparecen como iniciativa para identificar a los líderes digitales destacados durante cierto período por su nivel de influencia.

Para la elección de éstos se consideran cuatro ejes: 1) El eje de huella digital, se basa principalmente en métricas de datos duros que son generados por la dinámica de los influencers en redes sociales a través de su contenido y el engagement que acumulan por su presencia en las redes. Los indicadores que miden el impacto de la influencia son followers, likes, views, shares, exposición, entre otros. 2) Los players de la industria son individuos involucrados en el área comercial tales como empresarios, directores de marca, expertos en marketing, que establecen la perspectiva sobre los influencers y su valor para la empresa a nivel comercial y estratégico. 3) El comité Eliot establece el perfil del influencer de acuerdo a su alcance, relevancia de contenido y crecimiento en la industria. Esto mediante el procesamiento de los datos de la dinámica de los influencers a través de analítica y datos cualitativos. Y 4) El comité de influencers, son líderes digitales con una amplia carrera en la industria que apoyan con su conocimiento sobre la calidad y valor del contenido que ofrecen los concursantes (Eliot-Awards, 2019).

Por su parte, los influencers son personas que cuentan con conocimiento especializado en el contenido que generan para su comunidad. El contenido debe generar impacto entre los seguidores y usuarios de internet mediante el mensaje que transmiten y obtener un nivel alto de atención pública. La Tabla 1 muestra los influencers reconocidos por los premios Eliot en 2019, 2018 y 2020, y su actual número de seguidores en Facebook, Twitter, Instagram y YouTube.

Tabla 1

Influencers

\begin{tabular}{|c|c|c|c|}
\hline Cat & 2018 & 2019 & 2020 \\
\hline \multirow[t]{5}{*}{ Storyteller } & Juca & TV Ana Emilia & Skabeche \\
\hline & $\begin{array}{l}\text { 1,8 M seguidores (JUCA, } \\
\text { 2020a) }\end{array}$ & $\begin{array}{l}58 \mathrm{~K} \text { seguidores (Emilia } \\
\text { Contreras Oficial, 2020) }\end{array}$ & $\begin{array}{l}\text { 1,186 M seguidores } \\
\text { (SKabeche, 2020a) }\end{array}$ \\
\hline & $\begin{array}{l}\text { 5,68 M suscriptores } \\
\text { (JUCA, 2020b) }\end{array}$ & $\begin{array}{l}\text { 10,9 M suscriptores (TV Ana } \\
\text { Emilia, 2020) }\end{array}$ & $\begin{array}{l}\text { 9.89 M suscriptores (Más } \\
\text { SKabeche , 2020) }\end{array}$ \\
\hline & $\begin{array}{l}\text { 2,9 M seguidores (Papi } \\
\text { JUCA, 2020) }\end{array}$ & - & $\begin{array}{l}\text { 996 K seguidores } \\
\text { (SKabeche, 2020b) }\end{array}$ \\
\hline & $\begin{array}{l}4 \text { M seguidores } \\
\text { (jucaviapri, 2020) }\end{array}$ & $\begin{array}{l}\text { 1,626 M seguidores } \\
\text { (tv_ana_emilia, 2020) }\end{array}$ & - \\
\hline \multirow[t]{2}{*}{ How to } & Mis Pastelitos & Cristina Dacosta & Julio Profe \\
\hline & $\begin{array}{l}\text { 2,317 M seguidores (Mis } \\
\text { Pastelitos , 2020a) }\end{array}$ & $\begin{array}{l}744 \text { K seguidores (Cristina } \\
\text { Dacosta, 2020a) }\end{array}$ & $\begin{array}{l}\text { 515,6 K (julioprofenet, } \\
\text { 2020) }\end{array}$ \\
\hline
\end{tabular}




\begin{tabular}{|c|c|c|c|c|}
\hline & $\nabla$ & $\begin{array}{l}\text { 11,4 M suscriptores (Mis } \\
\text { Pastelitos, 2020b) }\end{array}$ & $\begin{array}{l}\text { 3,41 M suscriptores } \\
\text { (Dacosta'sBakery, 2020) }\end{array}$ & $\begin{array}{l}\text { 4,31 M suscriptores } \\
\text { (julioprofe, 2020) }\end{array}$ \\
\hline & & $\begin{array}{l}\text { 378,5 K seguidores (Gris } \\
\text { Verduzco, 2020) }\end{array}$ & $\begin{array}{l}\text { 679,2Kseguidores (Cristina } \\
\text { Dacosta, 2020b) }\end{array}$ & - \\
\hline & Co & $\begin{array}{l}\text { 4,5 M seguidores } \\
\text { (mispastelitos, 2020) }\end{array}$ & $\begin{array}{l}\text { 2,7 M seguidores } \\
\text { (cristinadacosta, 2020) }\end{array}$ & $\begin{array}{l}\mathbf{2 4 0} \mathrm{K} \text { seguidores (Julio } \\
\text { Ríos, 2020) }\end{array}$ \\
\hline \multirow{5}{*}{ Comedia } & & Ricardo O Farril & Lonrot & La cotorrisa \\
\hline & & $\begin{array}{l}\text { 1,051 M seguidores } \\
\text { (Ricardo O'Farrill, 2020a) }\end{array}$ & $\begin{array}{l}\text { 4,7 M seguidores (Lonrot, } \\
\text { 2020a) }\end{array}$ & \multirow[t]{4}{*}{$\begin{array}{l}\text { Se premia la serie de } \\
\text { videos. }\end{array}$} \\
\hline & & - & $\begin{array}{l}5.8 \mathrm{M} \text { suscriptores (Lonrot, } \\
2020 \mathrm{~b})\end{array}$ & \\
\hline & & $\begin{array}{l}\text { 560,4 K seguidores } \\
\text { (Ricardo O'Farrill, 2020b) }\end{array}$ & - & \\
\hline & & $\begin{array}{l}844 \text { K seguidores } \\
\text { (richieofarrill, 2020) }\end{array}$ & $\begin{array}{l}\text { 1,2 M seguidores (lonrot, } \\
2020 \text { ) }\end{array}$ & \\
\hline \multirow[t]{5}{*}{ Migrador } & & Juanpa Zurita & N/A & $\mathrm{N} / \mathrm{A}$ \\
\hline & & $\begin{array}{c}\text { 6,3 M seguidores } \\
\text { (Juanpa Zurita, 2020a) }\end{array}$ & & \\
\hline & & $\begin{array}{c}\text { 10,5 M seguidores } \\
\text { (Juanpa Zurita, 2020b) }\end{array}$ & & \\
\hline & & $\begin{array}{c}\text { 7,6 M seguidores } \\
\text { (JUANPA ZURITA, 2020) }\end{array}$ & & \\
\hline & & $\begin{array}{l}\text { 25,2 M suscriptores } \\
\text { (juanpazurita, 2020) }\end{array}$ & & \\
\hline \multirow{5}{*}{$\begin{array}{l}\text { Revelación del } \\
\text { año }\end{array}$} & & Calle y Poché & Yolo & Fede Vigevani \\
\hline & & $\begin{array}{l}\text { 4.8 } \mathrm{M} \text { seguidores (Calle } \\
\text { \& Poche, 2020) }\end{array}$ & $\begin{array}{l}541 \mathrm{~K} \text { seguidores (Yolo, } \\
2020 \mathrm{a} \text { ) }\end{array}$ & $\begin{array}{l}\text { 607,7 K seguidores } \\
\text { (Federico Vigevani, 2020a) }\end{array}$ \\
\hline & & $\begin{array}{l}\text { 7,55 M suscriptores } \\
\text { (Calle y Poché , 2020a) }\end{array}$ & $\begin{array}{l}12,3 \mathrm{M} \text { suscriptores (Yolo, } \\
2020 \mathrm{~b})\end{array}$ & $\begin{array}{l}\mathbf{8 , 2 3} \mathbf{M} \text { suscriptores (Fede } \\
\text { Vigevani, 2020b) }\end{array}$ \\
\hline & & $\begin{array}{l}\text { 2,2 M seguidores (Calle y } \\
\text { Poché, 2020b) }\end{array}$ & - & - \\
\hline & & $\begin{array}{l}\text { 5,2 M seguidores } \\
\text { (calleypoche, 2020) }\end{array}$ & $\begin{array}{l}\text { 3,2 M seguidores (YOLO, } \\
\text { 2020c) }\end{array}$ & - \\
\hline \multirow[t]{5}{*}{ Líder digital } & & Luisito Comunica & Beth Oh! & Kimberly Loaiza \\
\hline & & $\begin{array}{l}\text { 12,3 M seguidores } \\
\text { (Luisito Comunica, } \\
\text { 2020a) }\end{array}$ & $\begin{array}{l}\text { 1,3 M seguidores (Juan } \\
\text { Bertheau - Berth, 2020) }\end{array}$ & $\begin{array}{l}\text { 6,6 M seguidores (Kimberly } \\
\text { Loaiza, 2020a) }\end{array}$ \\
\hline & $\triangleright$ & $\begin{array}{l}\text { 34,3 M suscriptores } \\
\text { (Luisito Comunica, } \\
\text { 2020b) }\end{array}$ & $\begin{array}{l}\text { 9,25 M suscriptores (BERTH } \\
\mathrm{OH} !, 2020 \text { ) }\end{array}$ & $\begin{array}{l}\text { 26,3 M suscriptores } \\
\text { (Kimberly Loaiza, 2020b) }\end{array}$ \\
\hline & & $\begin{array}{l}8 \mathrm{M} \text { seguidores (Luisillo } \\
\text { El Pillo, 2020) }\end{array}$ & $\begin{array}{l}\text { 1,5 M seguidores } \\
\text { (Berthungas 5000, 2020) }\end{array}$ & $\begin{array}{l}\text { 5,3 M seguidores (Kimberly } \\
\text { Loaiza , 2020c) }\end{array}$ \\
\hline & 0 & $\begin{array}{l}\text { 22,3 M seguidores } \\
\text { (luisitocomunica, 2020) }\end{array}$ & $\begin{array}{l}\text { 5,1 M seguidores } \\
\text { (juanbertheau, 2020) }\end{array}$ & $\begin{array}{l}\text { 23,6 M seguidores } \\
\text { (kimberly.loaiza, 2020) }\end{array}$ \\
\hline \multirow{5}{*}{$\begin{array}{l}\text { Esports } \\
\text { (Gamers y } \\
\text { Ciberatletas) }\end{array}$} & & Robleis & Mikeleo & Leza \\
\hline & & $\begin{array}{l}\mathbf{1 , 4} \text { M } \quad \text { seguidores } \\
\text { (RobleisIUTU, 2020a) }\end{array}$ & - & - \\
\hline & & $\begin{array}{l}\mathbf{1 3 , 2} \text { M suscriptores } \\
\text { (RobleisIUTU, 2020b) }\end{array}$ & $\begin{array}{l}112 \text { K suscriptores (MKleo, } \\
\text { 2020) }\end{array}$ & - \\
\hline & & $\begin{array}{l}794,2 \quad K \quad \text { suscriptores } \\
\text { (Robleis, 2020) }\end{array}$ & $\begin{array}{l}\text { 190,4 K suscriptores (T1 I } \\
\text { MkLeo, 2020) }\end{array}$ & $\begin{array}{l}\text { 13,2 K seguidores (Leza, } \\
2020)\end{array}$ \\
\hline & 0 & $\begin{array}{l}\mathbf{2 , 7} \quad \mathbf{M} \quad \text { seguidores } \\
\text { (robleisiutu, 2020) }\end{array}$ & $\begin{array}{l}59 \mathrm{~K} \text { seguidores (mkleosb, } \\
2020 \text { ) }\end{array}$ & - \\
\hline \multirow[t]{3}{*}{ Better U } & & $\mathrm{N} / \mathrm{A}$ & Martha Debayle & Paty Jordan \\
\hline & 占 & & $\begin{array}{l}\text { 2,8 M seguidores (Martha } \\
\text { Debayle, 2020a) }\end{array}$ & $\begin{array}{l}\text { 3,6 M seguidores (GYM } \\
\text { VIRTUAL, 2020) }\end{array}$ \\
\hline & $\nabla$ & & $\begin{array}{l}\text { 1,19 M suscriptores (Martha } \\
\text { Debayle, 2020b) }\end{array}$ & $\begin{array}{l}\mathbf{1 0 , 8} \mathbf{M} \quad \text { suscriptores } \\
\text { (gymvirtual, 2020) }\end{array}$ \\
\hline
\end{tabular}




\begin{tabular}{|c|c|c|c|c|}
\hline & ह & & $\begin{array}{l}\mathbf{2 , 4} \quad \mathbf{M} \quad \text { seguidores } \\
\text { (marthadebayle, 2020a) }\end{array}$ & - \\
\hline & 0 & & 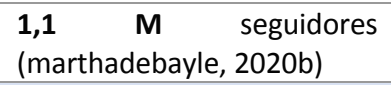 & $\begin{array}{l}\mathbf{1 , 4} \quad \mathbf{M} \quad \text { seguidores } \\
\text { (patryjordan, 2020) }\end{array}$ \\
\hline Beat for like & & $\mathrm{N} / \mathrm{A}$ & $N / A$ & JD Pantoja \\
\hline & 羊 & & & $\begin{array}{l}\text { 9,8 M seguidores (Juan De } \\
\text { Dios Pantoja Corona, 2020) }\end{array}$ \\
\hline & $\triangleright$ & & & $\begin{array}{l}\text { 10,3 M suscriptores (JD } \\
\text { Pantoja, 2020) }\end{array}$ \\
\hline & E & & & $\begin{array}{l}\text { 4,2 M seguidores (Juan De } \\
\text { Dios Pantoja, 2020) }\end{array}$ \\
\hline & 0 & & & 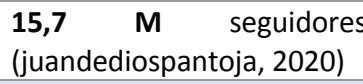 \\
\hline Networks & & $\mathrm{N} / \mathrm{A}$ & Badabun & $\mathrm{N} / \mathrm{A}$ \\
\hline & 卧 & & $\begin{array}{l}\text { 46,6 M seguidores } \\
\text { (Badabun, 2020a) }\end{array}$ & \\
\hline & $\triangleright$ & & $\begin{array}{l}\mathbf{4 3 , 7} \quad \mathbf{M} \quad \text { suscriptores } \\
\text { (Badabun, 2020b) }\end{array}$ & \\
\hline & 乡 & & $\begin{array}{l}396,7 \quad K \quad \text { seguidores } \\
\text { (Badabun, 2020c) }\end{array}$ & \\
\hline & 0 & & $\begin{array}{l}\mathbf{1 4 , 1} \mathbf{M} \text { seguidores } \\
\text { (badabun, 2020d) }\end{array}$ & \\
\hline
\end{tabular}

Fuente: elaboración propia con información de los Eliot-Awards (2019)

*N/A representa a las categorías que no fueron premiadas en el período considerado.

झ Facebook

$\triangleright$ YouTube

$\Leftrightarrow$ Twitter

(0) Instagram

Los datos duros, presentados previamente, muestran la tendencia de los principales líderes digitales y su perfil dinámico en las plataformas con más uso en México. Se observa que el influencer no tiene el mismo rendimiento en todas los canales. Por lo cual, una estrategia de IMKT debe elegir la plataforma correcta para cumplir con los objetivos de la empresa. Existen otros parámetros estadísticos de estas plataformas digitales, tales como: reacciones, alcance de la publicación, número de visitas al canal, número de reproducción de videos, comentarios y veces que se menciona o hace referencia. La combinación de estos parámetros permite lograr campañas exitosas de IMKT, así como establecer la remuneración económica de los influencers que prescriben la marca (Forbes, 2019).

Entre los casos de éxito de campañas de IMKT (informe de Eliot-Awards, 2019) está "No te hagas güey," patrocinada por Cerveceros de México bajo la colaboración del líder de opinión Luisito Comunica, logró un alcance de influencia de 4,483,273 personas. Asimismo, la organización dedicada al Influencer Marketing, Influencity, reporta el impulso de un producto de la marca Havaianas que tuvo la colaboración de 50 instagramers, quienes se dividieron en dos grupos. Cada uno contaba con compromisos de publicidad diferentes y de esta manera se logró obtener un alcance potencial de 1.8 millones de usuarios, 140, 000 likes, 6600 cometarios entre la comunidad y niveles de compromiso, sin precedentes, del 8.5 \%, (Influencity, 2019).

Los influencers perciben ingresos de manera proporcional al alcance de sus cifras e impacto que tienen en la comunidad. Por ejemplo, la monetización que obtienen de YouTube por la popularidad de un video, es decir por las visitas registradas. El cálculo se traduce en costo por clic, donde aproximadamente por cada 1000 clics a los videos, en México los youtubers ganan 5 pesos (El Universal, 2018). Esto a gran escala permite cuantificar los ingresos que por video un influencer puede percibir. Según el portal SocialBlade se tiene estimado que un 
influencer como Luisito Comunica tiene ingresos de $\$ 265.8 \mathrm{~K}$ a $\$ 4.3 \mathrm{M}$ USD. Juanpa Zurita va de $\$ 18.1 \mathrm{~K}$ a $\$ 290.2 \mathrm{~K}$ USD (Social Blade LLC, 2019).

Sin embargo, no todas las organizaciones cuentan con los recursos económicos suficientes para contratar este tipo de influencers. Por lo que las empresas optan por figuras con menores cifras de impacto que puedan ajustarse a sus necesidades. Plataformas como Coobis, Influencity, Internet República, entre otras, aparecen en el mercado como una de las formas de colaborar con los influencers que se adecuan al presupuesto de las organizaciones. Estas plataformas fungen como intermediarias entre influencers o generadores de contenido y empresas donde se evalúan métricas mediante algoritmos y softwares para evaluar las necesidades de la campaña y el perfil del influencer.

En el caso de las micro, pequeñas y medianas empresas, la inversión de las aplicaciones del IMKT llegan a percibirse fuera de su alcance. No obstante, una alternativa viable es recurrir a la influencia ya creada de sus clientes, colaboradores y proveedores para la difusión de su mensaje mediante advocacy programs. Esta práctica implica la reafirmación de la fidelidad de un consumidor o colaborador con la marca. La utilización de las plataformas digitales y redes sociales resulta ser un impulso en la difusión de la empresa (El Universal, 2018).

También existen opciones de empresas dedicadas a llevar a cabo el proceso que permite incentivar esta adopción de estrategias de IMKT en pequeñas y medianas empresas como es SocialPubli.com y SocialReacher.com. Ambas tienen como objetivo hacer crecer a aquellos seguidores que transmitan satisfacción y los convierte en embajadores de las marcas, pues son catalogados como los mejores prescriptores y motivan efectividad (Social Blade LLC, 2019).

Otra de las prácticas a las que recurren las empresas para poner en práctica el WOM son los patrocinios, donde el personaje de influencia se ve orientado a una prescripción a la empresa. En esta práctica los protagonistas no necesariamente tienen presencia en las redes sociales o son expertos en algún campo. La monetización está inclinada alrededor de la imagen y alcance de la fama (Photoslurp, 2019). En este sentido, los influencers pueden ser prescriptores de la marca, pero dejarían de lado la parte orgánica de los comentarios, situación que puede ser perjudicial para el influencer en caso de no hacer pública esta situación (Gómez Nieto, 2018).

La industria de los deportes aparece como gran escenario para el patrocinio, la cual es una de las más rentables en el panorama internacional. Según Photoslurp (2019), existen plataformas, como Hootkit, que se encargan de medir, a través de los rankings, la capacidad de impacto que tiene las figuras que están inclinadas a la prescripción de marcas multinacionales como Adidas, Nike, LAFC, Maclaren, New Balance, entre otras. Las figuras más rentables del deporte catalogadas por Hootkit (2019) se muestran en la tabla 2, donde destaca el deporte del football.

Tabla 2

Suscriptores a hootkit para patrocinio

\begin{tabular}{ccccccc}
\hline \multirow{2}{*}{ Rank } & Nombre & Deporte & Seguidores & Nuevos & Posteos & Interacciones \\
\hline 1 & Lionel Messi & Global Football & $200.9 \mathrm{M}$ & $2.5 \mathrm{M}$ & 45 & $53.0 \mathrm{M}$ \\
\hline 2 & Conor McGregor & Mixed Martial Arts & $45.8 \mathrm{M}$ & $313.2 \mathrm{~K}$ & 121 & $42.3 \mathrm{M}$ \\
\hline 3 & Kylian Mbappe & Global Football & $31.5 \mathrm{M}$ & $1.3 \mathrm{M}$ & 221 & $37.2 \mathrm{M}$ \\
\hline 4 & Neymar da Silva & Global Football & $216.1 \mathrm{M}$ & $1.6 \mathrm{M}$ & 78 & $36.8 \mathrm{M}$ \\
\hline 5 & Sergio Ramos & Global Football & $70.0 \mathrm{M}$ & $742.0 \mathrm{~K}$ & 80 & $24.9 \mathrm{M}$ \\
\hline
\end{tabular}

Fuente: elaboración propia con adaptaciones de Hootkit (2019) 


\subsection{IMKT frente a la pandemia del SARS-CoV-2}

De acuerdo con los resultados publicados en la ENDUITH (Encuesta Nacional sobre Disponibilidad y Uso de Tecnologías de la Información en los Hogares) se estima que de 2018 a 2019 se dio un crecimiento de 4.3 puntos porcentuales en el número de usuarios de internet en México. Lo cual, equivale a 80.6 millones de personas, de los cuales el $87.8 \%$ lo utilizan para tener acceso a las redes sociales (INEGI, 2020; SCT, 2020; IFT, 2020). Se espera que este aumento sea más significativo en 2020, toda vez que a partir de marzo de este año las acciones del gobierno federal para evitar la propagación del virus SARS-CoV-2, llevó a que la población tuviera que habitar espacios digitales principalmente en plataformas de reuniones como Zoom, Cisco Web Meetings, Google Meet, Google Meets, entre otras, que apoyan a desarrollar actividades en el trabajo y educación.

Uno de los principales impactos en los medios digitales se vio reflejado en el e-commerce. Según el reporte Impacto del Covid-19 en Venta Online publicado por la Asociación Mexicana de Venta Online (Online, 2020) las compras por tiendas electrónicas tuvieron un aumento en México del $60 \%$ en el trimestre de abril a julio del 2020. Este crecimiento provocó que las empresas se integrarán de forma acelerada al comercio electrónico, razón por la cual también parte de sus mecanismos como el marketing se inclinaron hacia las redes sociales y los medios digitales.

Las empresas están poniendo atención a una nueva generación de influencers de la plataforma Tiktok y consumidores con un nuevo estilo de vida. Por lo que, el cambio de estrategia de marketing es inevitable y necesario. Ante un panorama de decrecimiento económico, las empresas están ajustando sus presupuestos en IMKT y se podrían inclinar por perfiles específicos de influencers que tienen mayor impacto, aunque no estén catalogados en un alcance macro (Rincón, 2020).

\section{Conclusiones}

Las empresas requieren adoptar modelos de negocios que integren actividades que les creen y capturen valor para todas las partes interesadas. Las estrategias del IMKT a través de la extracción de datos de fuentes externas, influencer, impactan en el tráfico y fidelización de clientes, proveedores, colaboradores, etcétera, y esto a su vez en los rendimientos económicos de la empresa.

Las organizaciones para lograr los resultados esperados de la implementación del IMKT, deben determinar las características del Influencer, la plataforma digital correcta y los parámetros de rendimiento en función de tiempo y costos. Plataformas como Coobis, Influencity e Internet República aparecen como una de las formas de colaborar con los influencers ajustándose a las necesidades y presupuesto de las organizaciones. Para el caso de pequeñas empresas SocialPubli.com y SocialReacher.com buscan incentivar la aplicación de estas estrategias de IMKT.

La literatura revisada indica que los comentarios sobre marcas en el contenido de los influencers perciben un grado de naturaleza orgánica. Sin embargo, las marcas adquieren ciertos compromisos con los influencers. Esto implica la monetización del contenido y comentarios positivos que persiguen influenciar el comportamiento del consumidor. Es decir, se utilizan prácticas de patrocinio y se deja de lado la parte orgánica de la opinión, como lo establece el WOM. Por lo que resulta importante establecer mecanismos que garanticen la independencia de los influencers respecto a las empresas involucradas.

La confianza contenida en las fuentes externas para extraer valor, las tecnologías disponibles que dan soporte al procesamiento de la información y los costos que se generan, son los principales retos de las organizaciones para integrar las estrategias del IMKT en sus modelos de negocio. Finalmente, se tiene una nueva generación de influencers de la plataforma Tiktok. 


\section{Referencias bibliográficas}

Baber, A., Thurasamy, R., Malik, M. I., Sadiq, B., Islam, S., y Sajjad, M. (2016). Online word-of-mouth antecedents, attitude and intention-to-purchase electronic products in Pakistan. Telematics and Informatics, 33(2), 388-400. https://doi.org/10.1016/j.tele.2015.09.004

Badabun. (2020a). Número de seguidores Facebook. Recuperado el 13 de noviembre de 2020 de https://www.facebook.com/BadabunOficial

Badabun. (2020b). Número de seguidores YouTube. Recuperado el 13 de noviembre de 2020 de https://www.youtube.com/user/badabunOficial

Badabun. (2020c). Número de seguidores Twitter. Recuperado el 13 de noviembre de 2020 de https://twitter.com/BadabunOficial

badabun. (2020d). Número de seguidores Instagram. Recuperado el 13 de noviembre de 2020 de https://www.instagram.com/badabun/

Balaji, M. S., Khong, K. W., y Chong, A. Y. L. (2016). Determinants of negative word-of-mouth communication using social networking sites. Information and Management, 53(4), 528-540. https://doi.org/10.1016/j.im.2015.12.002

Bamakan, S. M. H., Nurgaliev, I., y Qu, Q. (2019). Opinion leader detection: A methodological review. Expert Systems with Applications, 115, 200-222.

Berlanga, R., García-Moya, L., Nebot, V., Aramburu, M. J., Sanz, I., y Llidó, D. M. (2015). Slod-Bi. International Journal of Data Warehousing and Mining, 11(4), 1-28. https://doi.org/10.4018/ijdwm.2015100101

BERTH OH! (2020). Número de seguidores YouTube. Recuperado el 13 de noviembre de 2020 de https://www.youtube.com/channel/UC2IDkGu61j_mwNJOc54Sb5w

Berthungas 5000. (2020). Número de seguidores Twitter. Recuperado el 13 de noviembre de 2020 de https://twitter.com/JuanBertheau

Bharadwaj, A., Sawy, O. A. El, Pavlou, P. A., y Venkatraman, N. (2013). Digital Business Strategy: Toward a Next Generation of Insights. MIS Quarterly, 27(3), 425-478.

Božič, K., y Dimovski, V. (2019). Business intelligence and analytics for value creation: The role of absorptive capacity. International Journal of Information Management, 46(November 2018), 93-103. https://doi.org/10.1016/j.ijinfomgt.2018.11.020

Calle \& Poche. (2020). Número de seguidores Facebook. Recuperado el 13 de noviembre de https://www.facebook.com/CalleYPoche

Calle y Poché . (2020a). Número de seguidores YouTube. Recuperado el 13 de noviembre de https://www.youtube.com/channel/UC-BZEmUUUIXmD1CgkWACVyQ

Calle y Poché. (2020b). Número de seguidores Twitter. Recuperado el 13 de noviembre de https://twitter.com/CalleyPoche

calleypoche. (2020). Número de seguidores Instagram. Recuperado el 13 de noviembre de https://www.instagram.com/calleypoche/ 
Cristina Dacosta. (2020a). Número de seguidores Facebook. Recuperado el 13 de noviembre de https://www.facebook.com/dacostasbakery

Cristina Dacosta. (2020b). Número de seguidores Twitter. Recuperado el 13 de noviembre de https://twitter.com/dacostacristina

cristinadacosta. (2020). Número de seguidores Instagram. Recuperado el 13 de noviembre de https://www.instagram.com/cristinadacosta/

Cui, R., Gallino, S., Moreno, A., y Zhang, D. J. (2018). The Operational Value of Social Media Information. Production and Operations Management, 27(10), 1749-1769. https://doi.org/10.1111/poms.12707

Dacosta'sBakery. (2020). Número de seguidores YouTube. Recuperado el 13 de noviembre de https://www.youtube.com/channel/UCOoqvyaHSH0_eBpP7pFj9qg

El Universal. (2018). ¿Cúanto ganan los youtubers mexicanos? Recuperado de https://www.eluniversal.com.mx/de-ultima/cuanto-ganan-los-youtubers-mexicanos

Eliot-Awards. (2019). Eliot Mediacom. Recuperado de https://eliotmedia.mx/

Emilia Contreras Oficial. (2020). Número de seguidores Facebook. Recuperado el 13 de noviembre de https://www.facebook.com/emiliacontrerasoficial

Fede Vigevani. (2020b). Número de seguidores YouTube. Recuperado el 13 de noviembre de https://www.youtube.com/user/fedexvige

Federico Vigevani. (2020a). Número de seguidores Facebook. Recuperado el 13 de noviembre https://www.facebook.com/fedevigevani

Forbes. (2019). Amazon busca nuevos influencers para pagarles hasta 28,500 pesos. Recuperado de https://www.forbes.com.mx/amazon-busca-nuevos-influencers-para-pagarles-hasta-28500-pesos/

Gómez Nieto, B. (2018). El influencer: herramienta clave en el contexto digital de la publicidad engañosa. Methaodos. Revista De Ciencias Sociales, 6(1), 149-156. https://doi.org/10.17502/m.rcs.v6i1.212

Gris Verduzco. (2020). Número de seguidores Twitter. Recuperado el 13 de noviembre de https://twitter.com/mis_pastelitos

GYM VIRTUAL. (2020). Número de seguidores Facebook. Recuperado el 13 de noviembre de https://www.facebook.com/gymvirtual

gymvirtual. (2020). Número de seguidores YouTube. Recuperado el 13 de noviembre de https://www.youtube.com/user/gymvirtual

Haryani, S., y Motwani, B. (2015). Discriminant model for online viral marketing influencing consumers behavioural intention. Pacific Science Review B: Humanities and Social Sciences, 1(1), 49-56. https://doi.org/10.1016/j.psrb.2015.12.002

Hootkit. (2019). Hootkit. Recuperado de https://www.hookit.com/

Influencity. (2019). Las 5 campañas con influencers más exitosas de Instagram. Recuperado de https://influencity.com/blog/es/campanas-exitosas-en-instagram/ 
JD Pantoja. (2020). Número de seguidores YouTube. Recuperado el 13 de noviembre de https://www.youtube.com/channel/UCrEsfMzcv3zFZkaSj8jsB2g

Juan Bertheau - Berth. (2020). Número de seguidores Facebook. Recuperado el 13 de noviembre de 2020 de https://www.facebook.com/JuanBertheau

Juan De Dios Pantoja Corona. (2020). Número de seguidores Facebook. Recuperado el 13 de noviembre de https://www.facebook.com/JuanDeDiosPantojaCorona

Juan De Dios Pantoja. (2020). Número de seguidores Twitter. Recuperado el 13 de noviembre de https://twitter.com/Juandedios_P

juanbertheau. (2020). Número de seguidores Instagram. Recuperado el 13 de noviembre de https://www.instagram.com/juanbertheau/

juandediospantoja. (2020). Número de seguidores Instagram. Recuperado el 13 de noviembre de https://www.instagram.com/juandediospantoja/

JUANPA ZURITA. (2020). Número de seguidores Twitter. Recuperado el 13 de noviembre https://twitter.com/JuanpaZurita

Juanpa Zurita. (2020a). Número de seguidores Facebook. Recuperado el 13 de noviembre de https://www.facebook.com/ElJuanpaZurita

Juanpa Zurita. (2020b). Número de seguidores YouTube. Recuperado el 13 de noviembre de youtube.com/channel/UCUo7T81zvCqpPhfsPb_kajA

juanpazurita. (2020). Número de seguidores Instagram. Recuperado el 13 de noviembre de https://www.instagram.com/juanpazurita/

JUCA. (2020a). Número de seguidores Facebook. Recuperado el 13 de noviembre de https://www.facebook.com/jucaviapri

JUCA. (2020b). Número de seguidores YouTube. Recuperado el 13 de noviembre de https://www.youtube.com/c/jucaviapri/channels

jucaviapri. (2020). Número de seguidores Instagram. Recuperado el 13 de noviembre de https://www.instagram.com/jucaviapri/

Julio Ríos. (2020). Número de seguidores Instagram. Recuperado el 13 de noviembre de https://www.instagram.com/julioprofenet/

julioprofe. (2020). Número de seguidores YouTube. Recuperado el 13 de noviembre de https://www.youtube.com/user/julioprofe/videos

julioprofenet. (2020). Número de seguidores Facebook. Recuperado el 13 de noviembre de https://www.facebook.com/julioprofenet

Kimberly Loaiza. (2020a). Número de seguidores Facebook. Recuperado el 13 de noviembre de https://www.facebook.com/kimberlyloaizaa

Kimberly Loaiza. (2020b). Número de seguidores YouTube. Recuperado el 13 de noviembre de https://www.youtube.com/channel/UCQZfFRohQ7UX-0CdXI-6pwQ 
Kimberly Loaiza. (2020c). Número de seguidores Twitter. Recuperado el 13 de noviembre de https://twitter.com/KimberlyLoaiza_

kimberly.loaiza. (2020). Número de seguidores Instagram. Recuperado el 13 de noviembre de https://www.instagram.com/kimberly.loaiza/

Kotler, P., y Keller, K. L. (2012). Dirección de Marketing. (Decimocuar). México: PEARSON EDUCACIÓN.

Leza. (2020). Número de seguidores Twitter. Recuperado el 13 de noviembre de https://twitter.com/leza_lol

Li, F., y Du, T. C. (2011). Who is talking? An ontology-based opinion leader identification framework for wordof-mouth marketing in online social blogs. Decision Support Systems, 51(1), 190-197.

https://doi.org/10.1016/j.dss.2010.12.007

Lin, H. C., Bruning, P. F., y Swarna, H. (2018). Using online opinion leaders to promote the hedonic and utilitarian value of products and services. Business Horizons, 61(3), 431-442. https://doi.org/10.1016/j.bushor.2018.01.010

Liu, S., Jiang, C., Lin, Z., Ding, Y., Duan, R., y Xu, Z. (2015). Identifying effective influencers based on trust for electronic word-of-mouth marketing: A domain-aware approach. Information Sciences, 306, 34-52. https://doi.org/10.1016/j.ins.2015.01.034

lonrot. (2020). Número de seguidores Instagram. Recuperado el 13 de noviembre de https://www.instagram.com/lonrot/

Lonrot. (2020a). Número de seguidores Facebook. Recuperado el 13 de noviembre de facebook.com/lonrot

Lonrot. (2020b). Número de seguidores YouTube. Recuperado el 13 de noviembre de https://www.youtube.com/user/lonrot

Luisillo El Pillo. (2020). Número de seguidores Twitter. Recuperado el 13 de noviembre de https://twitter.com/LuisitoComunica

Luisito Comunica. (2020a). Número de seguidores Facebook. Recuperado el 13 de noviembre de https://www.facebook.com/LuisitoComunica

Luisito Comunica. (2020b). Número de seguidores YouTube. Recuperado el 13 de noviembre de https://www.youtube.com/c/luisitocomunica/videos

luisitocomunica. (2020). Número de seguidores Instagram. Recuperado el 13 de noviembre de https://www.instagram.com/luisitocomunica/

Martha Debayle. (2020a). Número de seguidores Facebook. Recuperado el 13 de noviembre de https://www.facebook.com/DebayleOficial

Martha Debayle. (2020b). Número de seguidores YouTube. Recuperado el 13 de noviembre de https://www.youtube.com/user/DebayleMartha

marthadebayle. (2020a). Número de seguidores Twitter. Recuperado el 13 de noviembre de https://twitter.com/marthadebayle

marthadebayle. (2020b). Número de seguidores Instagram. Recuperado el 13 de noviembre de https://www.instagram.com/marthadebayle/ 
Más SKabeche. (2020). Número de seguidores YouTube. Recuperado el 13 de noviembre de https://www.youtube.com/channel/UCOn5Ihqix8mEqGsjygiJk6Q

Mis Pastelitos. (2020a). Número de seguidores Facebook. Recuperado el 13 de noviembre de https://www.facebook.com/mispastelitostutoriales

Mis Pastelitos. (2020b). Número de seguidores YouTube. Recuperado el 13 de noviembre de https://www.youtube.com/user/mispastelitosyoutub

mispastelitos. (2020). Número de seguidores Instagram. Recuperado el 13 de noviembre de https://www.instagram.com/mispastelitos/

MKleo. (2020). Número de seguidores YouTube. Recuperado el 13 de noviembre de https://www.youtube.com/channel/UCVAR_BDFcIXJ1Q9ndUhWLCA

mkleosb. (2020). Número de seguidores Instagram. Recuperado el 13 de noviembre de https://www.instagram.com/mkleosb/

Muntean, M., Cabău, L. G., y Rînciog, V. (2014). Social Business Intelligence: A New Perspective for Decision Makers. Procedia - Social and Behavioral Sciences, 124, 562-567. https://doi.org/10.1016/j.sbspro.2014.02.520

Online. (2020). Reporte Impacto COVID-19 en Venta Online. Recuperado de https://www.amvo.org.mx/estudios/reporte-4-0-impacto-covid-19-en-venta-online-mexico/

Palacios-Marqués, D., Merigó, J. M., y Soto-Acosta, P. (2015). Online social networks as an enabler of innovation in organizations. Management Decision, 53(9), 1906-1920. https://doi.org/10.1108/MD-062014-0406

Papi JUCA. (2020). Número de seguidores Twitter. Recuperado el 13 de noviembre de https://twitter.com/jucaviapri

patryjordan. (2020). Número de seguidores Instagram. Recuperado el 13 de noviembre de https://www.instagram.com/patryjordan/

Petrescu, M., O'Leary, K., Goldring, D., y Ben Mrad, S. (2018). Incentivized reviews: Promising the moon for a few stars. Journal of Retailing and Consumer Services, 41(March 2017), 288-295. https://doi.org/10.1016/j.jretconser.2017.04.005

Photoslurp. (2019). ¿Qué es el Marketing de Influencers? Recuperado de https://hi.photoslurp.com/es/blog/que-es-el-marketing-de-influencers/

Porter, M. E. (1996). What is Strategy? Harvard Business Review 74(6), 61-78.

Qlik. (2019). Página oficial. Recuperado de https://www.qlik.com/es-es/

Ren, J., Yeoh, W., Shan Ee, M., y Popovič, A. (2018). Online consumer reviews and sales: Examining the chickenegg relationships. Journal of the Association for Information Science and Technology, 69(3), 449-460. https://doi.org/10.1002/asi.23967

Ricardo O'Farrill. (2020b). Número de seguidores Twitter. Recuperado el 13 de noviembre de https://twitter.com/richieofarrill_ 
Ricardo O'Farrill. (2020a). Número de seguidores Facebook. Recuperado el 13 de noviembre de https://www.facebook.com/richiesgenial1

richieofarrill. (2020). Número de seguidores Instagram. Recuperado el 13 de noviembre de https://www.instagram.com/richieofarrill/

Robleis. (2020). Número de seguidores Twitter. Recuperado el 13 de noviembre de https://twitter.com/RobleisIUTU

robleisiutu. (2020). Número de seguidores Instagram. Recuperado el 13 de noviembre de https://www.instagram.com/robleisiutu/

RobleisIUTU. (2020a). Número de seguidores Facebook. Recuperado el 13 de noviembre de https://www.facebook.com/RobleisIUTU

RobleisIUTU. (2020b). Número de seguidores YouTube. Recuperado el 13 de noviembre de https://www.youtube.com/user/RobleisIUTU

Saura, J. R., y Bennett, D. R. (2019). A three-stage method for data text mining: Using UGC in business intelligence analysis. Symmetry, 11(4). https://doi.org/10.3390/sym11040519

Sharma, R., Ahuja, V., y Alavi, S. (2018). The Future Scope of Netnography and Social Network Analysis in the Field of Marketing. Journal of Internet Commerce, 17(1), 26-45.

https://doi.org/10.1080/15332861.2017.1423533

Simon, K. (2019). DIGITAL 2019: Global Digital Yearbook. Recuperado de https://wearesocial.com/blog/2019/01/digital-2019-global-internet-use-accelerates

Sivarajah, U., Irani, Z., Gupta, S., y Mahroof, K. (2019). Role of big data and social media analytics for business to business sustainability: A participatory web context. Industrial Marketing Management. https://doi.org/https://doi.org/10.1016/j.indmarman.2019.04.005

SKabeche. (2020a). Número de seguidores Facebook. Recuperado el 13 de noviembre de https://www.facebook.com/SKabecheTV

SKabeche. (2020b). Número de seguidores Twitter. Recuperado el 13 de noviembre de https://twitter.com/SKabecheTV

Social Blade LLC. (2019). Youtube Stats Summary. Recuperado de https://socialblade.com/youtube/channel/UCUo7T81zvCqpPhfsPb_kajA

Sukrat, S., y Papasratorn, B. (2018). An architectural framework for developing a recommendation system to enhance vendors' capability in C2C social commerce. Social Network Analysis and Mining, 8(1), 1-13. https://doi.org/10.1007/s13278-018-0500-7

T1 | MkLeo. (2020). Número de seguidores Twitter. Recuperado el 13 de noviembre de https://twitter.com/Mkleosb

Tableau. (2019). Tableau. 5 ejemplos reales de inteligencia de negocios en acción. Recuperado de https://www.tableau.com/es-mx/learn/articles/business-intelligence-examples

Teece, D. J. (2007). Explicating dynamic capabilities: the nature and microfoundations of (sustainable). ENTERPRISE PERFORMANCE, 1350(February 2004), 1319-1350. https://doi.org/10.1002/smj 
Tsutsumi, D., y Silva, T. (2019). Identifying Virtual Relations among Businesses Exploring User Reaction on Facebook. Proceedings - 2018 IEEE/WIC/ACM International Conference on Web Intelligence, WI 2018, 96103. https://doi.org/10.1109/WI.2018.0-102

TV Ana Emilia. (2020). Número de seguidores YouTube. Recuperado el 13 de noviembre de https://www.youtube.com/c/TVAnaEmilia/featured

tv_ana_emilia. (2020). Número de seguidores Instagram. Recuperado el 13 de noviembre de https://www.instagram.com/tv_ana_emilia/

Uzunoğlu, E., y Misci Kip, S. (2014). Brand communication through digital influencers: Leveraging blogger engagement. International Journal of Information Management, 34(5), 592-602. https://doi.org/10.1016/j.ijinfomgt.2014.04.007

We Are Social. (2019). DIGITAL 2019: México. Recuperado de https://datareportal.com/reports/digital-2019mexico

YOLO. (2020). Número de seguidores Twitter. Recuperado el 13 de noviembre de https://www.instagram.com/medicenyolo/

Yolo. (2020a). Número de seguidores Facebook. Recuperado el 13 de noviembre de https://www.facebook.com/MeDicenYolo

Yolo. (2020b). Número de seguidores YouTube. Recuperado el 13 de noviembre de https://www.youtube.com/channel/UCQ8TuCvcDMepleXFyOQfyOQ

Yuan, H., Xu, W., Li, Q., y Lau, R. (2018). Topic sentiment mining for sales performance prediction in ecommerce. Annals of Operations Research, 270(1-2), 553-576. https://doi.org/10.1007/s10479-017-24217

Zengin Alp, Z., y Gündüz Öğüdücü, Ş. (2018). Identifying topical influencers on twitter based on user behavior and network topology. Knowledge-Based Systems, 141, 211-221.

https://doi.org/10.1016/j.knosys.2017.11.021

Zhu, Z. (2013). Discovering the influential users oriented to viral marketing based on online social networks. Physica A: Statistical Mechanics and Its Applications, 392(16), 3459-3469. https://doi.org/10.1016/j.physa.2013.03.035

Zott, C., y Amit, R. (2009). Innovación del modelo de negocio: creación de valor en tiempos de cambio. Universia Business Review, (23), 108-121.

Esta obra está bajo una Licencia Creative Commons Attribución-NoCommercial 4.0 International

\section{(cc) EY-NC}

